\title{
Comparison of Bit Error Rate Performance of different Modulation Techniques over Turbulent FSO Link
}

\author{
Harjeevan Singh \\ Assistant Professor, Department of ECE, \\ Chandigarh University \\ Gharuan, Punjab (India)
}

\author{
Mohit Arora \\ Assistant Professor, Department of ECE, \\ Chandigarh University \\ Gharuan, Punjab (India)
}

\begin{abstract}
Free space optical (FSO) communication link is a line of sight (LOS) wireless link between the nodes separated by an unguided medium such that visible and infrared bands of the spectrum are used as data carrier. Recent past has witnessed huge revival of research interest in this field so as to make FSO commercially viable and looking into this prospective, data modulation techniques are one of the primary ways to enhance link sustainability. In this paper, comparison of modulation techniques like M-ary QAM, BPSK and DPSK has been studied by creating turbulent atmospheric conditions using gamma-gamma turbulence model. During this link analysis, BPSK performed far better in comparison to DPSK and M-ary QAM in terms of BER for similar SNR values and interestingly, higher the order of M-QAM and higher the link range, greater was the degradation of link performance observed.
\end{abstract}

\section{General Terms}

Free Space Optics, Modulation Techniques, Bit Error Rate.

\section{Keywords}

FSO, BER, M-QAM, BPSK, DPSK, Gamma Gamma model,Link Range.

\section{INTRODUCTION}

A Free Space Optical Communication refers to line of sight communication link between the communicating nodes separated by atmosphere as unguided medium between them. A main requirement for FSO link is line-of-sight condition between the two networking locations. The data to be transmitted can be modulated using intensity, phase or frequency characteristics of the optical carrier beam which lies in the infrared (IR) and visible part of spectrum which corresponds to the wavelengths between $850 \mathrm{~nm}$ and 1550 $\mathrm{nm}[1]$.

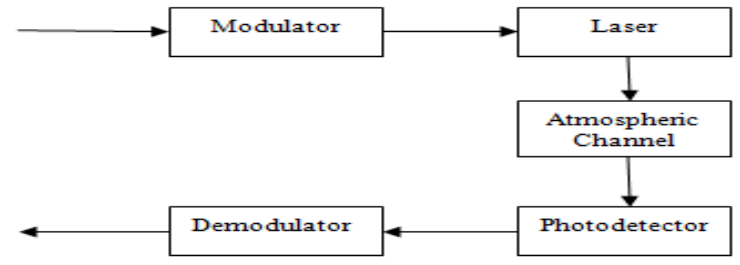

Fig. 1 Schematic of FSO

Traditionally, wireless technology has always been associated with radio frequency. But transmission by other carrier than RF waves can be more advantageous in some applications. The main advantage of FSO technology is the availability of huge bandwidth which could help in providing broadband wireless extensions to end-users. RF based wireless networks can offer data rates from tens of Mbps (point-to-multipoint) up to several hundred Mbps (point-to-point) whereas FSO Technology can provide date rates varying form hundred Mbps to several Gigabits[2] . Spectrum congestion in RF systems face an exponentional increase in bandwidth requirements around the globe and it is one of the biggest challenges in expansion of networks. The most efficient solution to this problem is the use of FSO system which guarantees abundant bandwidth because with an optical carrier, bandwidth of upto $2000 \mathrm{THz}$ can be delivered, which is as high as $10^{5}$ of current RF networks. Miscellaneous features like data security, licence free spectrum, quick and easy set up of link, no digging of trenches etc. have made FSO need of the hour. Moreover the cost of FSO system is also lower than RF systems[3].

The greatest challenge that FSO faces result from the atmospheric channel, resulting in signal scattering, absorption and fluctuation[4]. The travelling beam through atmosphere comes across huge attenuation due to which a huge amount of data loss takes place. The atmospheric channel attenuates the field traversing it as a result of absorption and scattering processes. The major challenge facing the deployment of FSO systems is their performance in different weather conditions, particularly in the presence of fog. The principal disadvantage of using such communication systems is the harmful effect of the atmosphere on light transmission and thus on the link availability and reliability. The severity and duration of these effects will affect the allowable distance and the availability of the links. The main factor responsible for FSO performance degradation is the atmospheric scintillation caused by atmospheric temperature inhomogeneity[5]. Scintillation results in signal fading which degrades the system performance. Scintillation is caused by the variation in temperature due to which zones of differing density act as lenses and light gets scattered from its intended path. Thus, the interest in this technology has led to the need to understand the effects of various weather conditions on laser radiation propagation in the atmosphere. There are various atmospheric models which have been suggested for understanding turbulent nature of atmosphere but gammagamma model is very effective in terms of predicting the atmospheric turbulence [6].

In this paper, BER analysis of BPSK, DPSK and M-ary QAM has been done for turbulent atmospheric conditions using gamma-gamma turbulent model while the rest of the paper is organised as follows: Section two contains description of the gamma-gamma turbulent model while modulation techniques and their available bit error rates expressions have been discussed in section three. Section four contains results and their related discussions about the different modulation techniques which are followed by conclusion in section five. 

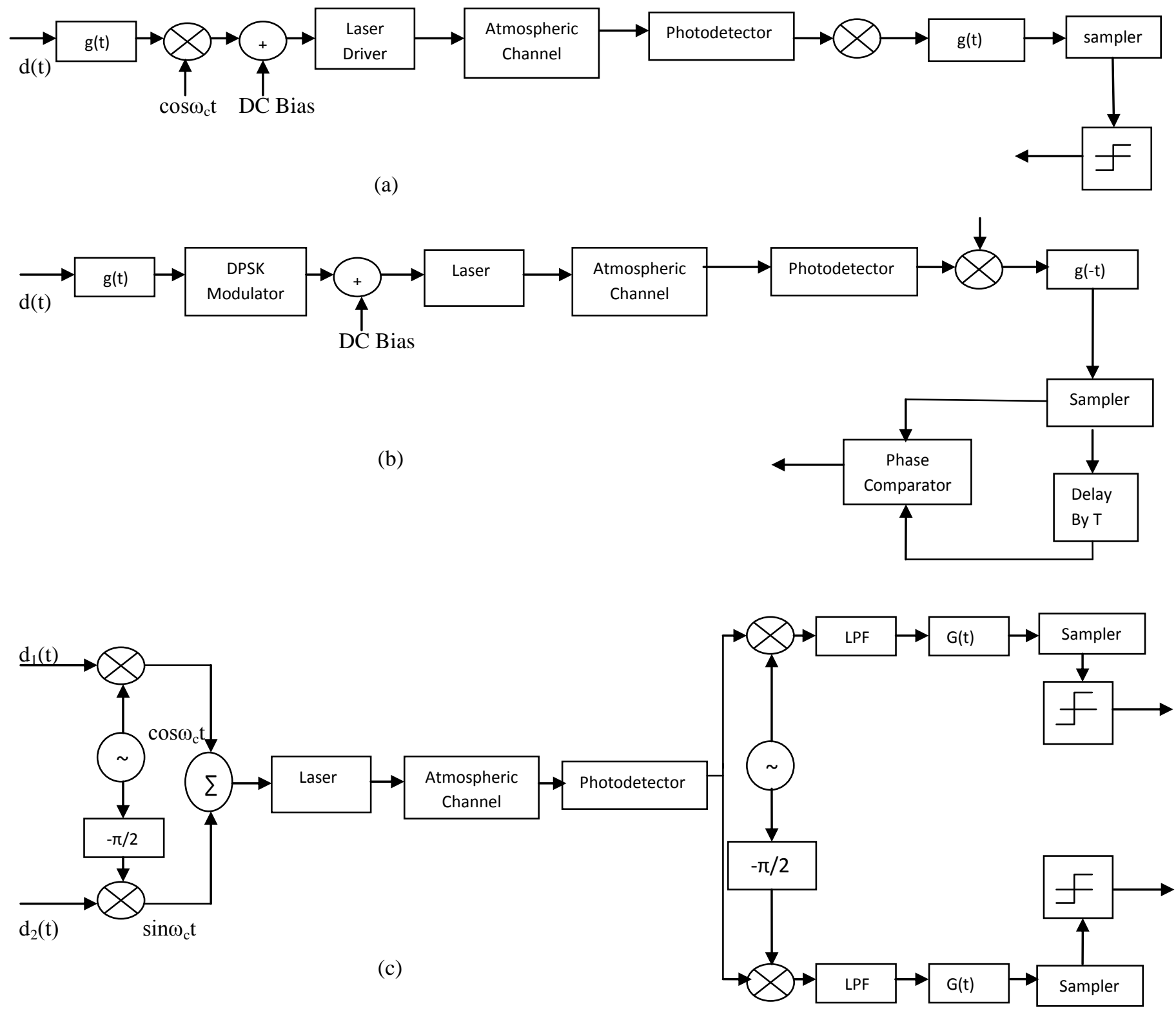

Fig. 2 FSO Link employing (a) BPSK Modualtion (b) DPSK Modulation (c) QAM Modulation

\section{THE GAMMA GAMMA}

\section{TURBULENCE MODEL}

The gamma-gamma turbulence model proposed by Andrews et al.[7] has been used to study the turbulent behaviour of atmosphere. This model is suitable for both weak and strong turbulence regimes. Large scale and small scale fading effects have been studied to predict the fluctuation of light radiation travelling turbulent atmosphere where it is assumed that large scale eddies modulate small scale eddies. The normalized received irradiance is given as the product of two random processes as follows:

$$
I=I_{x} I_{y}
$$

Where $I_{x}$ and $I_{y}$ arise from large scale and small scale eddies respectively and both of them are supposed to obey gamma gamma distribution.

The gamma gamma model for the probability density function of received irradiance is given by:

$$
P(I)=\frac{2(\alpha \beta)^{\frac{(\alpha+\beta)}{2}}}{\Gamma(\alpha) \Gamma(\beta)} I^{\frac{\alpha+\beta}{2}-1} K_{\alpha-\beta}(2 \sqrt{\alpha \beta I)}, \mathrm{I}>0
$$

Where $I$ is the received signal intensity, $\alpha$ and $\beta$ are parameters of the probability density function, $\Gamma$ is the gamma function and $K_{\alpha-\beta}$ is the modified bessel function of the second kind of order. Here, $\alpha$ and $\beta$ are the effective number of small-scale and large scale eddies of turbulent environment. These parameters are given as:

$$
\begin{aligned}
& \alpha=\left\{\exp \left[0.49 \sigma^{2} /\left(1+1.11 \sigma^{12 / 5}\right)^{7 / 6}\right]-1\right\}^{-1} \\
& \beta=\left\{\exp \left[0.51 \sigma^{2} /\left(1+1.11 \sigma^{12 / 5}\right)^{5 / 6}\right]-1\right\}^{-1}
\end{aligned}
$$

where $\sigma^{2}=1.23 \mathrm{C}_{\mathrm{n}}^{2} \mathrm{k}^{7 / 6} \mathrm{~L}^{11 / 6}$ is the rytov variance which represents the variance of log-intensity fluctuation in which $C_{n}^{2}$ is the refractive-index structure parameter, $k$ is the wave number, and $L$ is the distance between transmitter and receiver.

\section{MODULATION TECHNIQUES}

\subsection{BPSK Modulation}

BPSK is the simplest form of phase shift keying (PSK) which uses two different phases for data modulation such that phases 
are 180 degrees apart and hence it can also be termed as 2PSK. The general form for BPSK is as follows:

$$
s(t)=\sqrt{\frac{2 E_{b}}{T_{b}}} \cos \left(2 \pi f_{c} t+\pi(1-n)\right), n=0,1
$$

For subcarrier BPSK modulation, the output electrical signal is given by[8]:

$r(t)=I(t)+m I(t)\left[s_{i}(t) \cos \left(2 \pi f_{c} t\right)-s_{q}(t) \sin \left(2 \pi f_{c}(t)\right]+n_{i}(t) \times\right.$

$$
\cos \left(2 \pi f_{c} t\right)-n_{q}(t) \sin \left(2 \pi f_{c} t\right)
$$

where $s_{i}(t)$ and $s_{q}(t)$ are in phase and quadrature signals,respectively amd $m$ is the modulation index, $f c$ is the intermediate carrier frequency, $n_{i}(t)$ and $n_{q}(t)$ are the guassian processes with the variance $\sigma_{n}^{2}$. By taking into account the above factors, the conditional PDF $p(r \mid x)$ of received signal can be written as :

$$
\begin{gathered}
p(r \mid x)=\frac{2}{\sqrt{2 \pi} \sigma_{n} \Gamma(\alpha) \Gamma(\beta)}\left(\frac{\alpha \beta}{m}\right)^{\frac{\alpha+\beta}{2}} \int_{0}^{\infty} t^{\frac{\alpha+\beta}{2}-1} K_{\alpha-\beta}\left(2 \sqrt{\frac{\alpha \beta t}{m}}\right) \times \\
\exp \left[\frac{(r-t)^{2}}{2 \sigma_{n}^{2}}\right] \mathrm{dt}, \mathrm{x}=+1 \\
p(r \mid x)=\frac{2}{\sqrt{2 \pi} \sigma_{n} \Gamma(\alpha) \Gamma(\beta)}\left(\frac{\alpha \beta}{m}\right)^{\frac{\alpha+\beta}{2}} \int_{0}^{\infty} t^{\frac{\alpha+\beta}{2}}-1 K_{\alpha-\beta}\left(2 \sqrt{\frac{\alpha \beta t}{m}}\right) \times \\
\exp \left[\frac{(r+t)^{2}}{2 \sigma_{n}^{2}}\right] \mathrm{dt}, \mathrm{x}=-1
\end{gathered}
$$

The BER for BPSK modulated FSO link in turbulent atmosphere can be given by:

$P_{e}=\frac{(\alpha \beta)^{\frac{(\alpha+\beta)}{2}}}{\Gamma(\alpha) \Gamma(\beta)} \int_{0}^{\infty} x^{\frac{\alpha+\beta}{2}-1} K_{\alpha-\beta}\left(2 \sqrt{\alpha \beta I)} \operatorname{erfc}\left[\frac{m x}{\sqrt{2} \sigma_{n}}\right] \mathrm{dx}\right.$

Considering AWGN system the expression for BER for BPSK system can be simplified to:

$$
P_{b p s k}=\frac{1}{2} \operatorname{erfc}\left(\sqrt{\frac{m^{2}}{2 \sigma_{n}^{2}}}\right)
$$

\subsection{DPSK Modulation}

In Differential Phase shift keying (DPSK), the demodulator determines the changes in the phase of the received signal rather than the phase itself. Since this technique depends on the difference between successive phases, it is termed as DPSK. It is more simpler as compared to ordinary PSK since there is no need for the demodulator to have a copy of the reference signal to determine the exact phase of the received signal. So this technique is very useful especially in case of degraded FSO links where when the phase estimation for the carrier demodulation is not possible. In FSO systems, the modulated carrier RF signal is used to modulate the irradiance of an optical carrier. After traversing the atmospheric channel, the photdetector receives the irradiance and generates photocurrent accordingly which is given by [9]:

$$
I(t)=R I(1+\beta m(t))+n(t)
$$

Where $I=I_{\text {peak }} / 2, I_{\text {peak }}$ is the peak received irradiance, $R$ is the photodetector responsivity, $\beta$ is modulation index, $\mathrm{m}(\mathrm{t})=\mathrm{A}(\mathrm{t}) \cos \left(\omega_{\mathrm{c}} \mathrm{t}+\theta\right), \mathrm{n}(\mathrm{t})$ is the additive noise. Since the subcarrier has been pre-modulated using DPSK and it has non-varying amplitude and $\beta$ has been normalized to unity. So peak amplitude becomes $\mathrm{A}(\mathrm{t})=\mathrm{A} \leq 1$. The electrical SNR per bit is given by[10]:

$$
S N R_{e}=A^{2} R^{2} I^{2} / 2 \sigma^{2}
$$

The conditional BER for the DPSK technique is given by:

$$
P_{e c}=0.5 \exp \left(-0.5 S N R_{e}\right)
$$

In the presence of atmospheric turbulence,the unconditional $\mathrm{BER}$ is given by:

$P_{e}=\frac{(\alpha \beta)^{\frac{(\alpha+\beta)}{2}}}{\Gamma(\alpha) \Gamma(\beta)} \int_{0}^{\infty} x^{\frac{\alpha+\beta}{2}-1} K_{\alpha-\beta}\left(2 \sqrt{\alpha \beta I)} \times\left(0.5 \exp \left(-0.5 \mathrm{SNR}_{\mathrm{e}}\right)\right) \mathrm{dx}\right.$

\subsection{QAM Modulation}

QAM modulation is a method of combining two amplitudemodulated signals into a single channel, thereby doubling the effective bandwidth. In QAM, there are two carriers,each having the same frequency but differing in phase by 90 degrees(one quarter of cycle). Mathematically, one of the signals can be represented by a sine wave, and the other by a cosine wave. The two modulated carriers are combined at the source of transmission and then the resulting modualted carrier is used to modualte the irradiance of an optical. At the destination, the carriers are separated, the data is extracted from each, and then the data is combined into the original modulating signal. In QAM, we use two double sideband (DSB) signals using carriers of same frequency but in phase quadrature. The two baseband signals used are $m_{1}(t)$ and $m_{2}(t)$ and the corresponding QAM signal is given by:

$$
\Phi_{Q A M}(t)=m_{l}(t) \cos \omega_{c}(t)+m_{2}(t) \sin \omega_{c}(t)
$$

Both the modulated signals occupy same band but the two signals can be separated at the receiver by synchronous detection using two local carriers in phase quadrature. Now we consider data symbols which comprise $\log _{2} M$ data symbols which have been mapped to one of the $M$ phases on each carrier signal $m(t)$. So the conditional BER expression for QAM is given by[11]:

$$
P_{e c}=\frac{2(1-1 / \sqrt{M)}}{\log _{2} M} \mathrm{Q} \sqrt{\frac{3 \log _{2} M(S N R)}{2(M-1)}}
$$

The unconditional BER for FSO link is obtained by averaging the conditional bit error rate over the atmospheric turbulence stastics which is given by:

$P e=\frac{(\alpha \beta)^{\frac{(\alpha+\beta)}{2}}}{\Gamma(\alpha) \Gamma(\beta)} \int_{0}^{\infty} x^{\frac{\alpha+\beta}{2}-1}\left(2 \sqrt{\alpha \beta I)}\left(\frac{2\left(1-\frac{1}{\sqrt{M}}\right)}{\log _{2} M}\right) \mathrm{Q}\left(\sqrt{\frac{3 \log _{2} M\left(S N R_{e}\right)}{2(M-1)}}\right)\right.$

$d x$

\section{SIMULATION RESULTS AND DISCUSSIONS}

The system described above has been simulated using matlab.The simulation parameters used are given below as :

Table 1. Simulaton Parameters

\begin{tabular}{|l|l|}
\hline Parameters & Value \\
\hline Bit Rate $\left(\mathrm{R}_{\mathrm{b}}\right)$ & $155 \mathrm{Mbps}$ \\
\hline Link Range & $1 \mathrm{Km}$ \\
\hline Responsivity & 1 \\
\hline Modulation Index & 1 \\
\hline
\end{tabular}




\begin{tabular}{|l|l|}
\hline Temperature & $300 \mathrm{~K}$ \\
\hline Optical Filter Bandwidth & $1 \mathrm{e}-3 \mu \mathrm{m}$ \\
\hline Receiver Field of view & 0.6 radian \\
\hline $\begin{array}{l}\text { Refractive Index Structure } \\
\text { Parameter, } C_{n}^{2}\end{array}$ & $0.75 \mathrm{e}-14 \mathrm{~m}^{-2 / 3}$ \\
\hline Load Resistance & $50 \Omega$ \\
\hline Boltzman's Constant & $1.38 \mathrm{e}-23 \mathrm{~J} / \mathrm{K}$ \\
\hline Electronic Charge & $1.602 \mathrm{e}-19 \mathrm{C}$ \\
\hline
\end{tabular}

Fig. 5 shows the BER performance of BPSK, DPSK, 16QAM and 64-QAM and it is clear that BPSK performs better than the other techniques for a turbulent FSO link. DPSK performs better than 16-QAM and 64-QAM. So BPSK modulation technique is giving the best performance among the other modulation techniques.

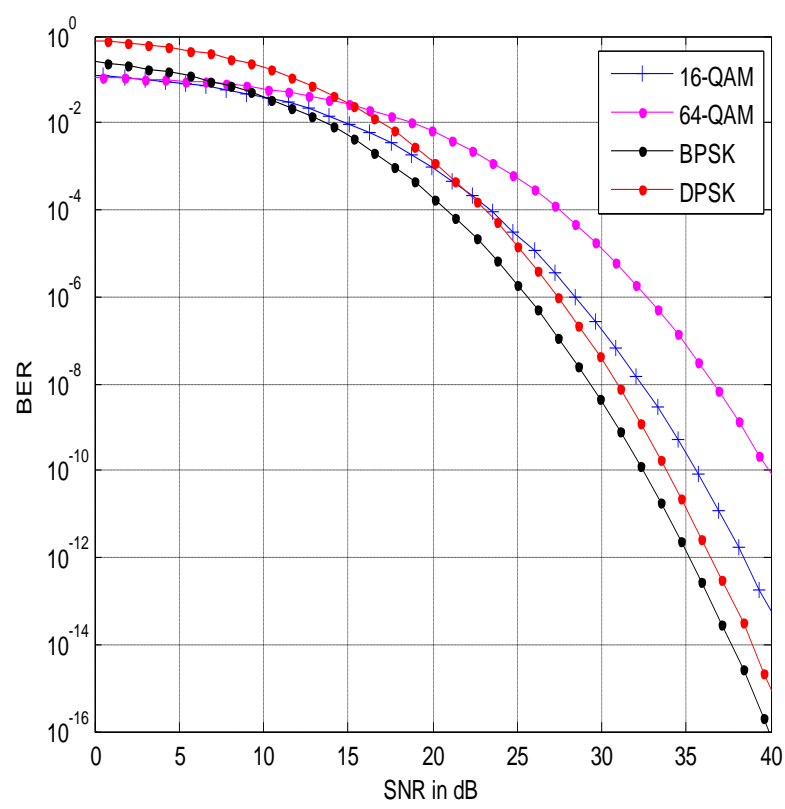

Fig. 5 BER versus SNR for different Modulation Technique

Fig. 3 Gamma-Gamma PDF for weak, moderate and strong turbulent regimes

Fig. 3 shows the probability density curve for gamma-gamma model with different values of turbulent strength. The values of alpha and beta indicate whether the atmospheric turblence region is weak, moderate or strong. In particular, the gammagamma model has a much higher denstiy in the high amplitude region leading to a more severe impact on the system performance.

Fig. 4 shows that BER performance of M-QAM for a FSO link. The BER has been plotted as a function of SNR for different values of $M$. It has been found that BER performance gets degraded as the value of $\mathrm{M}$ is increased. The BER performance of 64-QAM is highly degraded as compared to 8-QAM.

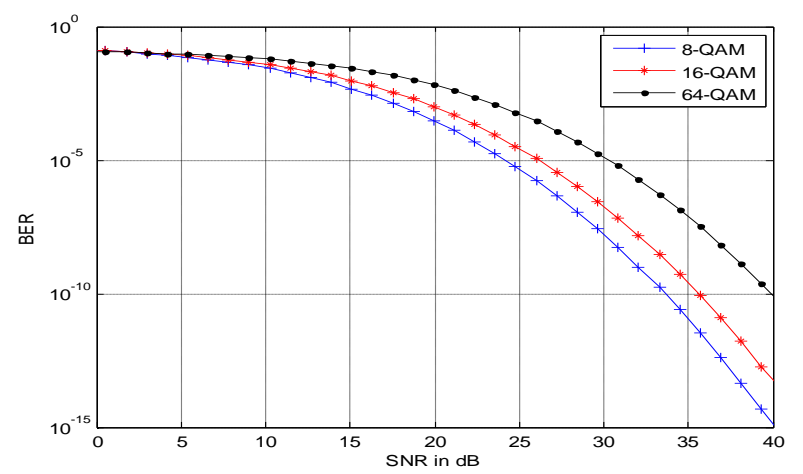

Fig. 4 BER versus SNR for M-QAM Modulation Technique

Fig. 6 shows BER performance of BPSK, DPSK, 8-QAM,16QAM and 64-QAM. BPSK is giving the best performance among the all techniques used for a FSO link but the BER performance of 8-QAM and DPSK becomes almost similar after SNR of $35 \mathrm{~dB}$.

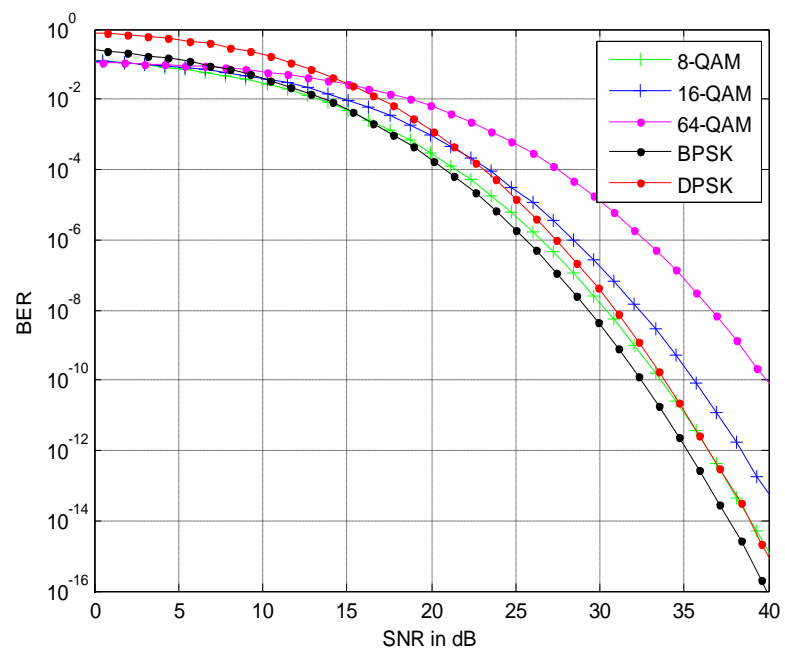

Fig. 6 BER versus SNR for different modulation techniques

Fig. 7 shows the BER perfromance of BPSK modulation technique for different link ranges. It has been observed that as the link range is getting increased, the BER performance is getting degraded. 


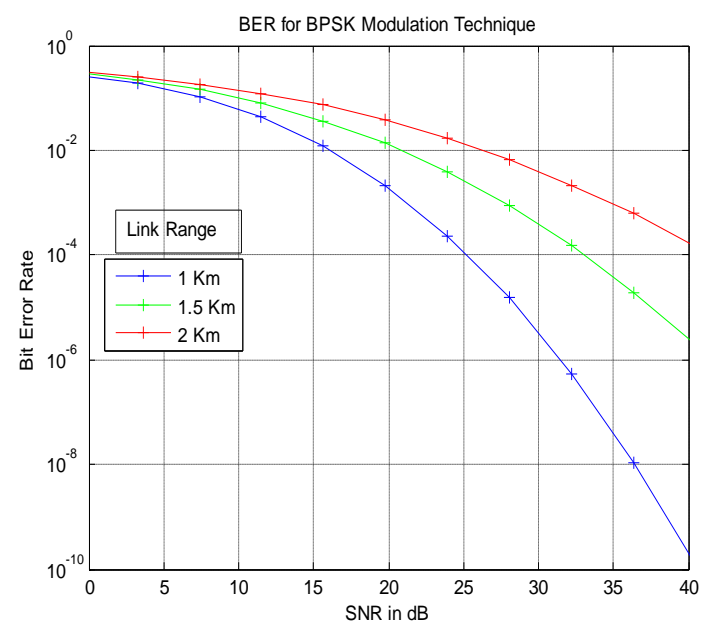

Fig. 7 BER versus SNR for BPSK using different link ranges

\section{CONCLUSIONS}

From the BER performance of BPSK, DPSK and M-QAM modulation techniques for a turbulent FSO link, we found that for higher order M-QAM, the BER performance of M-QAM degrades i.e. 16-QAM gives better BER performance than 64QAM. DPSK and 8-QAM behave more or less the similar but for higher values of $\mathrm{M}$ (16-QAM and 64-QAM) the performance of DPSK is far better. For lower values of SNR i.e. up to $15 \mathrm{~dB}$, the performance of all the modulation techniques is more or less the similar but for higher SNR values, BPSK modulation technique delivers better BER performance than the other discussed techniques. Moreover, the BER performance also gets degraded as the link range is increased.

\section{ACKNOWLEDGEMENT}

I would like to thank Mr. Ajaypaul Singh Channa, Mr. Sumit Sharma, Mr. Harpushap Singh, Mr. Prabhjot Singh and Mr. Umesh Gupta for their sincere help for the completion of this research work.

\section{REFERENCES}

[1] H. Willebrand and B.S Ghuman,Free Space Optocs: Enabling Optical Connectivity in today's network.Indianapolis:SAMS publishing,2002.

[2] Isaac I. Kim,Bruce McArthur, and E.Korevaar, "Comparison of laser beam propagation at $785 \mathrm{~nm}$ and $1550 \mathrm{~nm}$ in fog and haze for optical wireless communication,"SPIE Proceeding:Optical Wireless Communications III,vol. 4214,pp. 26-37,2001.

[3] D. Kedar and S. Arnon, "Optical wireless communication through fog in the presence of pointing errors,"Applied Optics,vol. 42,pp. 4946-4954,Aug. 2003.

[4] S. Karp,R.M. Gagliardi,S.E Moran,and L.B Stotts,Optical Channels:fibers,clouds, water and the atmosphere.New York:Plenum Press, 1988.

[5] I.I. Kim,M. Mitchell,and E.J Korevaar, "Measurement of scintillation for free-space laser communication at 785 $\mathrm{nm}$ and $1550 \mathrm{~nm}$,"Optical Wireless Communications II,Proceedings of SPIE,vol. 3850,pp. 49-52,1999

[6] D. Killinger, "Free space optics for laser communication through the air,"Opt. Photon. News,vol. 13,no. 3,pp. 3642, Oct. 2002

[7] L.C. Andrews,R.L. Phillips, and C.Y. Hopen,Laser beam scintillation with applications,Bellingham:SPIE,2001.

[8] W.O Popoola,Z.Ghassemlooy,and E. Leitgeb, "Freespace optical communication using subcarrier modulation in gamma-gamma atmospheric turbulence," in $9^{\text {th }}$ International Conference on Transparent Optical Networks(ICTON '07) Rome Italy,Vol. 3,pp. 156-16,2007.

[9] R.M Gagliardi and S. Karp,Optical Communications, $2^{\text {nd }}$ Edition ed. New York:John Wiley,1995.

[10] W.O. Popoola,Z. Ghassemblooy and E. Leitgeb, "BER Performance of DPSK Subcarrier Modulated Free Space Optics in fully Developed Speckle",IEEECSNDSP,Graz,Austria,pp. 273-277,23-25 July 2008.

[11]J.G Proakis,Digital Communications,New York:McGraw-Hill,2004. 